\title{
Mediastinite em cirurgia cardíaca: tratamento com epíploon
}

\author{
Dielson Teixeira SAMPAIO*, José Carlos Resende ALVES*, Aluísio Ferreira SILVA*, \\ Nílcio Cunha LOBO Jr. ${ }^{*}$, Danilo SIMÕES*, Willian FARIA*, \\ Angelo LOBATO*, Carlos Camilo Smith FIGUEROA*
}

RBCCV 44205-483

Sampaio D T, Alves J C R, Silva A F, Lobo Jr. N C, Simões D, Faria W, Lobato A, Figueroa C C S-Mediastinite em cirurgia cardíaca: tratamento com epíploon. Rev Bras Cir Cardiovasc 2000; 15 (1): 23-31.

RESUMO: Casuística e Métodos: Foram analisados 22 casos de mediastinite que ocorreram após 1006 operações cardíacas com esternotomia, realizadas de 1993 a 1998, no Hospital Felício Rocho em Belo Horizonte, Minas Gerais. A complicação ocorreu em 3,2\% dos coronariopatas, 3,1\% dos transplantados, 1,1\% dos valvares e não ocorreu em portadores de defeitos congênitos.

Resultados: O índice geral de mediastinite foi de 2,1\%. O microorganismo mais comumente responsável pela infecção foi o Staphylococcus aureus (39,1\%). Diversas modalidades terapêuticas para mediastinite foram utilizadas no período: drenagem simples, desbridamento e granulação, rotação de retalho de músculo peitoral e rotação do grande epíploon.

Conclusão: A rotação do retalho de grande epíploon, introduzida no Serviço em 1995 para tratamento dos casos de mediastinite, proporcionou excelentes resultados, não ocorrendo óbitos pela complicação nos últimos 2,5 anos.

DESCRITORES: Mediastinite, cirurgia. Procedimentos cirúrgicos cardíacos, efeitos adversos. Esterno, cirurgia. Retalhos cirúrgicos. Omento, transplante.

\section{INTRODUÇÃO}

A espécie humana sofre os efeitos danosos das infecções que ocorrem após a perda da integridade da pele, seja acidental ou planejada. Apesar do aperfeiçoamento de inúmeras técnicas, fármacos e equipamentos, as infecções cirúrgicas persistem, muitas vezes tirando vidas.

A cirurgia cardíaca não foge à regra. Apesar de ser sofisticado método de tratamento invasivo, observamos a ocorrência de quadros infecciosos gravíssimos que desafiam a competência da equipe cirúrgica e dos centros de tratamento cardiológico.

A abertura do esterno para possibilitar o acesso ao coração, introduzida por MILTON $(1,2)$, em 1897, e popularizada por JULIAN (3) et al., tem sido evitada sempre que possível. Lança-se mão de procedimentos cardiológicos invasivos com cateteres ou balões (4) e, ultimamente, de abordagens cirúrgicas através de reduzidas incisões torácicas com ou sem o auxílio de videotoracoscopia (5-11), com excelentes resultados.

A incidência de mediastinite nos procedimentos operatórios cardíacos em que se pratica esternotomia é pequena, variando de 0,4 a $5 \%(12,13)$. A mortalidade desta temível complicação, no entanto, é alta, variando de 14 a $47 \%$ (14). Além desta alta mortalidade, verifica-se considerável aumento dos custos do tratamento após esta complicação, quase triplicando estes valores $(15,16)$.

Os fatores de risco parecem ser muitos, podendo ser citados: associação de diabete melito e

Trabalho realizado no Hospital Felício Rocho. Belo Horizonte, MG, Brasil.

Recebido para publicação em setembro de 1999.

* Do Hospital Felício Rocho.

Endereço para correspondência: Dr. Dielson Teixeira Sampaio. Rua Timbiras, 3642, sala 601. Belo Horizonte, MG, Brasil. CEP: $30.140-062$. Tel. (031) 295-3568. Fax: (031) 295-3014. e-mail: dielson@africanet.com.br 
Sampaio D T, Alves J C R, Silva A F, Lobo Jr. N C, Simões D, Faria W, Lobato A, Figueroa C C S - Mediastinite em cirurgia cardíaca: tratamento com epíploon. Rev Bras Cir Cardiovasc 2000; 15 (1): 23-31.

revascularização do miocárdio utilizando as duas artérias torácicas internas ${ }^{(17,18)}$, uso indiscriminado de eletrocautério ${ }^{(19,20)}$, doença pulmonar obstrutiva crônica (21), obesidade, uso de drogas beta-adrenérgicas ${ }^{(22)}$, doença coronariana, duração da operação, tempo de CEC, reoperações por sangramento, ventilação prolongada, tabagismo, etc. Apesar de todos estes e outros fatores serem cuidadosamente analisados por vários autores (23-26) é surpreendente a falta de consenso. Ao que parece, cada Instituição tem seus "próprios fatores de risco".

Instalada a mediastinite após uma operação cardíaca, a situação é de absoluto desafio para as equipes cirúrgicas. Ao longo do tempo, têm-se despendido muito empenho e criatividade nas tentativas de tratamento. Técnicas de drenagem aberta, desbridamento amplo e irrigação fechada têm sido utilizadas (27-29). Ultimamente, com a colaboração de cirurgiões plásticos, reconstruções têm sido feitas, primárias ou seguindo a drenagem aberta ou ainda após irrigação fechada que falhou, assim como em infecções crônicas. Utilizam-se nestas reconstruções, retalhos musculares (peitoral, reto abdominal) e também o epíploon (30-38).

O objetivo deste trabalho é apresentar nossa casuística dos últimos 6 anos e informar o manejo que estamos adotando, ultimamente, nos pacientes que desenvolvem mediastinite após esternotomias para operações cardíacas.

\section{CASUÍSTICA E MÉTODOS}

Registramos a ocorrência de 22 infecções mediastinais após a realização de 1006 operações com esternotomia (Tabela 1). A taxa global de mediastinite foi, portanto, de 2,1\%.

Não houve mediastinite em pacientes submetidos a retrocas valvares. Também não registramos mediastinite em pacientes submetidos à revasculari- zação miocárdica utilizando-se as duas artérias torácicas internas (21 operações). Em um dos pacientes submetidos à revascularização miocárdica foi realizada substituição da valva mitral concomitantemente. O perfil pré-operatório dos pacientes que desenvolveram mediastinite está representado na Tabela 2.

Exames pré-operatórios, inclusive avaliação dentária e ginecológica nas mulheres, foram sempre realizados, na tentativa de descobrir processos infecciosos. Pacientes internados durante vários dias foram sempre encaminhados ao Serviço de Controle de Infecção Hospitalar para medidas necessárias de descolonização de pele e mucosas antes da operação. A tricotomia com máquina sempre foi feita no momento da operação e, em todos pacientes, administramos antibiótico profilático (Cefalotina $2 \mathrm{~g}$ a cada 2 horas durante a operação e depois mais 3 doses). Uma serra vibratória a ar comprimido ou elétrica foi utilizada nas esternotomias. O uso de campos plásticos estéreis e aderentes sempre foi exigido.

As operações foram realizadas, em sua maioria, com circulação extracorpórea.

Alguns parâmetros per-operatórios dos pacientes que desenvolveram mediastinite estão exibidos na Tabela 3.

O diagnóstico de mediastinite foi feito através do acompanhamento clínico rigoroso no pós-operatório, com atenção absoluta para dor intensa e hiperemia na ferida operatória, febre (13 pacientes), leucocitose (15 pacientes), instabilidade do esterno, drenagem de secreções, piora das condições clínicas, derrames pericárdicos e pleurais. Exames complementares como radiografia de tórax, tomografia, ecocardiografia, mostrando derrames e coleções de líquido ou ar pré-esternais, assim como culturas de sangue, de cateteres e de drenos retirados, auxiliaram na investigação em casos suspeitos. Aspiração com agulha para Gram e cultura foi utilizada eventualmente. O tempo médio de aparecimento (feito o diagnóstico) foi de 12 dias (6 a 40 dias).

TABELA 1

INCIDÊNCIA DE MEDIASTINITE

\begin{tabular}{lccc}
\hline $\begin{array}{l}\text { OPERAÇÕES } \\
\text { REALIZADAS }\end{array}$ & $\begin{array}{c}\text { NÚMERO DE } \\
\text { PACIENTES }\end{array}$ & $\begin{array}{c}\text { NÚMERO DE } \\
\text { INFECTADOS }\end{array}$ & $\begin{array}{c}\text { ÍNDICE DE } \\
\text { INFECÇÃO(\%) }\end{array}$ \\
\hline RM & 523 & 17 & 3,2 \\
Valvar & 345 & 4 & 1,1 \\
Congênitos & 48 & -- & - \\
Transplantes & 32 & 1 & 3,1 \\
Outros & 58 & -- & -- \\
Total & 1006 & 22 & 2,1 \\
\hline
\end{tabular}

$\mathrm{RM}=$ revascularização miocárdica.

Outros = aneurismas, pericardiectomias. 
Sampaio D T, Alves J C R, Silva A F, Lobo Jr. N C, Simões D, Faria W, Lobato A, Figueroa C C S - Mediastinite em cirurgia cardíaca: tratamento com epíploon. Rev Bras Cir Cardiovasc 2000; 15 (1): 23-31.

TABELA 2

\begin{tabular}{lc}
\hline \multicolumn{2}{c}{ PERFIL PRÉ -OPERATÓRIO DOS PACIENTES QUE } \\
DESENVOLVERAM MEDIASTINITE \\
\hline Dados pré-operatórios \\
Idade média \\
Homens & 61,6 anos ( $23-78$ ) \\
Mulheres & $60 \%$ \\
Diabéticos & $40 \%$ \\
Obesos & $13 \%$ \\
Urgências & $52 \%$ \\
Reoperações & $13 \%$ \\
Infartos prévios & -- \\
Fração de ejeção & $39 \%$ \\
Dias de internação prévios & $51,6 \%(30-72)$ \\
\hline
\end{tabular}

TABELA 3

PARÂMETROS PER-OPERATÓRIOS DOS PACIENTES COM MEDIASTINITE

\section{PARÂMETROS PER-OPERATÓRIOS}

\begin{tabular}{ll}
\hline Tempo de CEC (média) & 67 minutos $(30-100)$ \\
Tempo de pinçamento aórtico & 53 minutos $(25-84)$ \\
Uso de artéria mamária & 13 pacientes \\
Transfusão de sangue & 21 pacientes \\
Uso de inotrópicos & 13 pacientes \\
Uso de CEC & 21 pacientes \\
\hline
\end{tabular}

CEC = circulação extracorpórea

Procedimentos como exploração por sangramento, drenagem pericárdica, traqueostomia, assim como, ocorrências de acidente vascular cerebral, derrame pleural, ventilação pulmonar prolongada e outros dados importantes na evolução destes pacientes infectados estão resumidos na Tabela 4 .

\section{TABELA 4}

INTERCORRÊNCIAS NO PERIOODO PÓS-OPERATÓRIO DE PACIENTES COM MEDIASTINITE

\begin{tabular}{lc}
\hline INTERCORRÊNCIAS & $\begin{array}{c}\text { NÚMERO DE } \\
\text { PACIENTES }\end{array}$ \\
\hline Acidente vascular cerebral & 6 \\
Ventilação prolongada (>24 h) & 8 \\
Derrame pleural & 7 \\
Consolidação pulmonar & 3 \\
Traqueostomia & 3 \\
Choque cardiogênico + BIA & 1 \\
Reoperação por sangramento & 2 \\
Drenagem pericárdica & 2 \\
\hline
\end{tabular}

$\mathrm{BIA}=$ balão intra-aórtico
TABELA 5

\begin{tabular}{lccc}
\hline \multirow{2}{*}{ FORMAS } & DE APRESENTAÇÃO & DAS MEDIASTINITES \\
\hline \multirow{2}{*}{ TIPO } & SUBTIPO & \multicolumn{2}{c}{ ESTERNO } \\
\cline { 3 - 4 } & & INSTÁVEL & ESTÁVEL \\
\hline \multirow{2}{*}{ Aguda } & segmentar & - & $2^{*}$ \\
& difusa & 15 & 4 \\
Crônica & segmentar & - & $2^{*}$ \\
& difusa & - & 1 \\
\hline \multirow{2}{*}{ * A tabela repete os 2 pacientes tratados posteriormente. }
\end{tabular}

Alguns pacientes apresentaram infecção em apenas uma região da ferida operatória (esternotomia), em outros esta era difusa.

O mesmo se deu quanto à instabilidade do esterno (deiscência), observada rapidamente em alguns pacientes. Noutros isto não ocorreu em nenhum momento. Na verdade, encontramos diferentes combinações de infecção: aguda ou crônica, segmentar ou difusa e a presença ou não de instabilidade do esterno.

Em 2 pacientes identificamos o que chamamos de mediastinite aguda segmentar. Em uma paciente, submetida a transplante cardíaco, a manifestação de mediastinite ocorreu 40 dias após o procedimento, a qual consideramos como crônica difusa.

Registramos a ocorrência de 4 casos de mediastinite aguda difusa, sem que houvesse instabilidade do esterno. A Tabela 5 mostra as formas de apresentação clínica das mediastinites.

Feito o diagnóstico de mediastinite, estes pacientes foram submetidos a algum tipo de tratamento, que foi diversificado ao longo destes 6 anos. A conduta terapêutica variou dependendo do quadro infeccioso e do estado clínico dos pacientes. Desta forma, identificamos três tipos de pacientes: no primeiro, os indivíduos apresentavam infecção aguda, macroscopicamente bem localizada em mediastino superior ou inferior (mediastinite aguda segmentar), com drenagem espontânea precoce e cursando com ótimo estado geral. No segundo, os pacientes apresentavam infecção aguda e difusa em todo o mediastino (mediastinite aguda difusa). O terceiro tipo esteve representado por apenas um caso, de uma paciente que apresentou mediastinite crônica difusa após transplante cardíaco.

O primeiro grupo (mediastinite aguda segmentar) foi tratado com drenagem ampla da ferida no ponto que demonstrava coleção e lavagens diárias com soro fisiológico e povidine tópico, fazendo-se curativos. Fios de sutura e outros fragmentos foram 
Sampaio D T, Alves J C R, Silva A F, Lobo Jr. N C, Simões D, Faria W, Lobato A, Figueroa C C S - Mediastinite em cirurgia cardíaca: tratamento com epíploon. Rev Bras Cir Cardiovasc 2000; 15 (1): 23-31.

retirados com o paciente em seu próprio leito. Antibióticos foram administrados de acordo com o microorganismo e sua sensibilidade.

O segundo grupo (mediastinite aguda difusa) foi encaminhado sistematicamente ao centro cirúrgico o mais rapidamente possível. Realizamos abertura do esterno e, após avaliação das condições locais e colheita de material para cultura, procedemos ao desbridamento exaustivo e lavagem das estruturas envolvidas. Nos pacientes em que a ferida operatória apresentava melhor aspecto macroscópico realizamos interposição imediata de retalho de grande epíploon (11 pacientes).

O retalho de epíploon foi feito por laparotomia supra-umbelical com descolamento do colo transverso e passagem pelo diafragma ou pela porção superior da laparotomia e via subcutâneo (Figura 1). Em uma paciente a liberação do epíploon foi realizada por laparoscopia. Em apenas 2 pacientes foi necessária ligadura e secção de um dos pedículos vasculares, o grande epíploon atingiu sem maiores dificuldades a porção superior da esternotomia infectada. A parede abdominal foi fechada de maneira habitual tomando-se os cuidados com o retaIho. Pele e subcutâneo foram sempre fechados e o esterno mantido aberto. Drenos tubulares para aspiração contínua foram colocados sistematicamente e mantidos por alguns dias até que a drenagem de secreções diminuísse.

Nos pacientes que apresentavam ferida com mal aspecto (muita necrose tecidual e grande quantidade de secreção purulenta) e más condições clínicas (choque) deixamos a mesma aberta após o desbridamento e os encaminhamos à UTI para suporte respiratório e circulatório, antibioticoterapia e curativos diários. Após alguns dias (média de 5 dias), realizamos transposição de algum tipo de retalho (músculo peitoral em 2 pacientes e grande epíploon em 5).

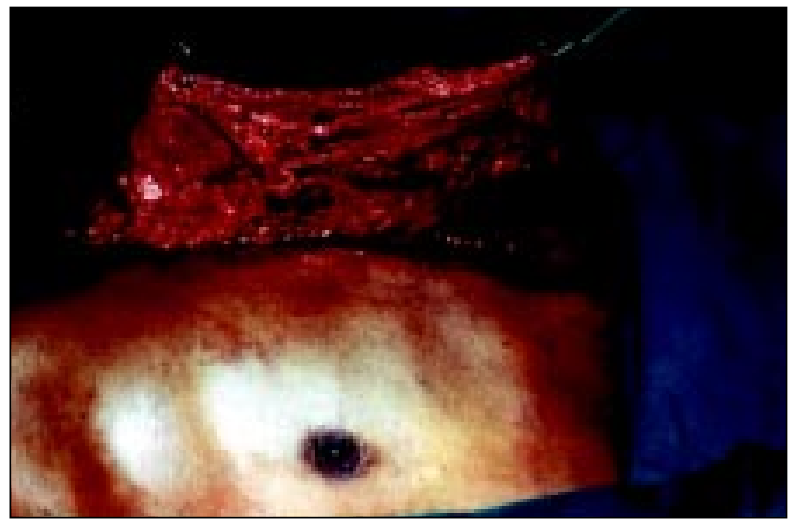

Fig. 1 - Rotação do retalho de epíploon.
O terceiro "grupo" esteve representado pela paciente transplantada que desenvolveu mediastinite crônica difusa, na qual realizamos desbridamento e interposição imediata do grande epíploon.

\section{RESULTADOS}

Tivemos índice de infecção (mediastinite) de $2,1 \%$. O Staphylococcus aureus foi o microorganismo mais freqüentemente isolado na ferida operatória de esternotomia $(39,1 \%)$, sendo em 4 oportunidades identificado como M.A.R.S.A. Registramos 17,3\% de infecção por Staphylococcus epidermidis. Os Gram negativos compareceram em menor freqüência. Na Tabela 6 estão apresentados os microorganismos encontrados nesta série.

A evolução dos pacientes tratados pelas diferentes técnicas pode ser vista na Tabela 7 .

Os 2 pacientes submetidos a drenagem simples e curativos diários desenvolveram fístulas crônicas.

Em duas oportunidades (pacientes graves e ferida com necrose acentuada e secreção purulenta) deixamos a ferida aberta para curativos diários na Unidade de Terapia Intensiva. Devido às péssimas condições clínicas estes pacientes faleceram antes de serem submetidos à rotação de retalho.

Os 2 pacientes submetidos a desbridamento, granulação e rotação de retalho de músculo peitoral faleceram.

Todos os 16 pacientes que foram submetidos à rotação de retalho de epíploon tiveram boa evolução. Em 5 deles a rotação do retalho foi feita após granulação da ferida e em 11 foi imediata, o resultado final de um desses casos pode ser observado na Figura 2.

Dos 22 pacientes com mediastinite, 18 sobreviveram (mortalidade geral de 18\%). Os óbitos ocorreram devido a sepse e falência de múltiplos ór-

TABELA 6

MICROORGANISMOS ISOLADOS NA FERIDA DE ESTERNOTOMIA

\begin{tabular}{lcr}
\hline MICROORGANISMO & $\begin{array}{c}\text { NÚMERO DE } \\
\text { VEZES }\end{array}$ & $\%$ \\
\hline Staphylococcus aureus $^{*}$ & 9 & 39,1 \\
Staphylococcus epidermides $^{\text {Pseudomonas }}$ & 4 & 17,3 \\
Acinetobacter calcoaceticos & 2 & 8,6 \\
Klebsiella sp. & 2 & 8,6 \\
Alcaligenes sp. & 1 & 4,3 \\
\hline
\end{tabular}

${ }^{*}$ M.A.R.S.A. em 4 oportunidades 
Sampaio D T, Alves J C R, Silva A F, Lobo Jr. N C, Simões D, Faria W, Lobato A, Figueroa C C S - Mediastinite em cirurgia cardíaca: tratamento com epíploon. Rev Bras Cir Cardiovasc 2000; 15 (1): 23-31.

TABELA 7

TIPOS DE TRATAMENTO PARA MEDIASTINITE E EVOLUÇÃO CLÍNICA

\begin{tabular}{lcc}
\hline TERAPIA UTILIZADA & NÚMERO DE PACIENTES & EVOLUÇÃO CLÍNICA \\
\hline Drenagem simples + curativos & 2 & Fístula crônica \\
Desbr. + granulação & 2 & Óbito \\
Desbr. + granulação + retalho de peitoral & 2 & Óbito \\
Desbr.+ granulação + retalho de epíploon & 5 & Boa \\
Desbr. + retalho imediato de epíploon & 11 & Boa \\
\hline Desbr. = desbridamento & & \\
\hline
\end{tabular}

gãos. Complicações graves que ocorreram em pacientes com mediastinite estão apresentadas na Tabela 8; algumas delas estiveram relacionadas com os óbitos.

Em 5 pacientes identificamos hérnias epigástricas que foram relatadas, mas quando questionados sobre dor ou outras queixas, as respostas foram sempre negativas.

Os 2 pacientes classificados como portadores

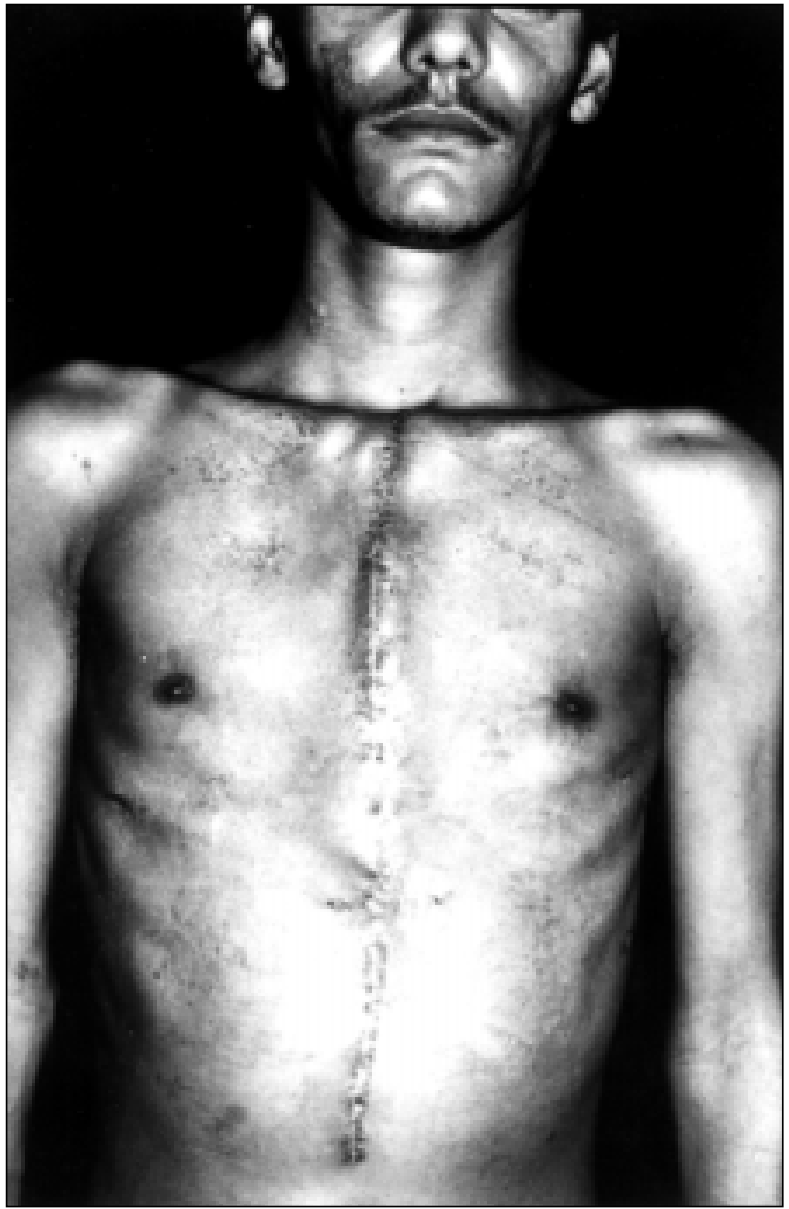

Fig. 2 - Aspecto final após rotação de retalho de epíploon. de mediastinite aguda segmentar e tratados conservadoramente desenvolveram fístula crônica na ferida (mediastinite crônica segmentar) e a cintilografia óssea demonstrou osteomielite de esterno. Procedeu-se ao mesmo processo de desbridamento e com rotação imediata do retalho de epíploon, com excelentes resultados.

\section{COMENTÁRIOS}

Se considerarmos que o índice de infecção em operações limpas do Hospital Felício Rocho é de $2,0 \%$ e, na literatura em torno de $1,9 \%{ }^{(39)}$, estamos nesta série com índice aceitável de 2,1\% para mediastinites. Naturalmente, estamos empenhados em melhorar este resultado.

Para o sucesso do tratamento da mediastinite é fundamental que o diagnóstico seja feito precocemente. Para isto um alto índice de suspeita precisa ser exercitado.

Infelizmente, a drenagem espontânea de secreções costuma demorar e o diagnóstico pode não ser fácil. Em nossa casuística, sinais como dor intensa na ferida (em dois casos com episódios de síncope), febre (em $56 \%$ dos casos), leucocitose em torno de 12.000 (69\% dos casos), instabilidade do esterno e recuperação pós-operatória arrastada com piora do estado geral, foram de grande valia no diagnóstico precoce.

TABELA 8

\begin{tabular}{lc}
\hline \multicolumn{2}{c}{ COMPLICAÇÕES OCORRIDAS EM PACIENTES } \\
PORTADORES DE MEDIASTINITE \\
\hline COMPLICAÇÕES & $\begin{array}{c}\text { NÚMERO DE } \\
\text { PACIENTES }\end{array}$ \\
\hline Insuficiência renal aguda & 9 \\
Insuficiência respiratória & 8 \\
Infecção pulmonar & 5 \\
Empiema do lado esquerdo & 4 \\
Hemorragia digestiva alta & 2 \\
\hline
\end{tabular}


Sampaio D T, Alves J C R, Silva A F, Lobo Jr. N C, Simões D, Faria W, Lobato A, Figueroa C C S - Mediastinite em cirurgia cardíaca: tratamento com epíploon. Rev Bras Cir Cardiovasc 2000; 15 (1): 23-31.

A tomografia computadorizada de tórax auxiliou em 6 casos, mostrando coleções retroesternais com níveis hidroaéreos em 5 pacientes e revelando osteomielite de esterno em 1 , como já relatado anteriormente.

Com relação ao aspecto da ferida operatória, deparamos ora com quadros de grande quantidade de secreção purulenta e muita necrose, ora com feridas relativamente limpas. Estamos intervindo precocemente, encontrando feridas com melhor aspecto, evitando assim quadros infecciosos adiantados e fazendo de imediato a rotação do grande epíploon. Com esta conduta estamos há quase 3 anos sem mortalidade por mediastinite (Gráfico 1).

O uso do grande epíploon em mediastinite foi inicialmente proposto por LEE et al. (36), em 1976, baseando-se em reconstruções da parede torácica descritas por KIRICUTA ${ }^{(40)}$, em 1963, e em antigas revascularizações miocárdicas ${ }^{(41)}$.

O grande epíploon, por sua eficiente propriedade em combater infecções, assim como sua reconhecida capacidade angiogênica ${ }^{(42)}$, capaz de suportar um enxerto de pele em sua superfície (43), aliada à facilidade de obtenção e à possibilidade de obliterar grandes espaços "mortos" nos impulsionou a utilizá-lo como retalho.

Os resultados têm sido compensadores. O que estamos fazendo ultimamente, diferente do que tem sido argumentado, é utilizar apenas um método de tratamento e em apenas um estágio, para tratar todos os tipos de mediastinite.

Desde a introdução em nosso Serviço da técnica de rotação do retalho de epíploon em 1995 , houve apenas 1 óbito por mediastinite. Este óbito ocorreu em paciente no qual se fez desbridamento e a ferida foi deixada aberta, devido ao mal estado clínico do paciente.

Devemos estar atentos a não utilizar o epíploon com eventuais metástases de tumores intra-abdominais, como alerta TELFER et al. (44).

A dor nestes pacientes, submetidos a repetidas operações e curativos diários, foi neutralizada

GRÁFICO 1

MEDIASTINITE E MORTALIDADE

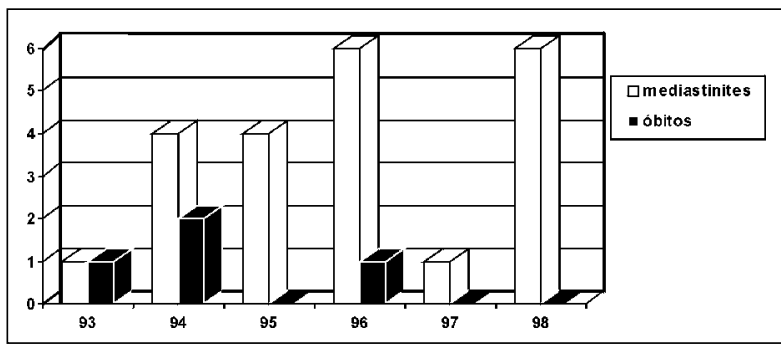

freqüentemente com analgesia controlada pelo próprio paciente (Patient-Controlled Analgesia). Este processo consiste na utilização de bombas injetoras programadas em que o próprio paciente aperta um botão e recebe uma dose limitada e suficiente de analgésico. Além do conforto no pós-operatório, que representa humanização no tratamento, outros benefícios importantes parecem advir desta técnica, incluindo: mobilização mais precoce, melhor cicatrização e recuperação funcional, menor ansiedade e medo, menor risco de trombose venosa profunda, de pneumonia e de infarto do miocárdio (45).

Talvez não seja demais insistir na questão infecção e deiscência já levantada por alguns autores $(46,47)$. De fato, ao longo de vários anos lidando com mediastinites, temos encontrado uma gama enorme de formas clínicas. Evidentemente, a presença de deiscência não significa que haja sempre infecção; encontramos casos de graves infecções do mediastino, sem deiscência. Na verdade, podemos encontrar deiscências segmentares ou totais, agudas ou crônicas. O mesmo se pode dizer das mediastinites. E todas estas combinações podem ocorrer.

Temos procurado utilizar estes parâmetros para definir o quadro clínico de cada paciente, evitando assim confusões com outras complicações de ferida, mais simples e facilmente solucionáveis como: fraturas e deiscências de esterno, abscessos de parede, seromas, enfisemas subcutâneos, etc.

Consideramos importantes algumas recomendações para evitar mediastinite:

- rigorosa avaliação pré-operatória do paciente em busca de focos infecciosos;

- preparo pulmonar prévio e interrupção do uso de cigarro;

- evitar dissecção das duas artérias torácicas internas em pacientes diabéticos;

- tempo de internação hospitalar mínimo antes da operação, evitando assim colonização do paciente por microorganismo altamente selecionados em virulência e resistência a antibacterianos. Se necessário, fazer banhos pré-operatórios com soluções anti-sépticas ou fazer "descolonização" de orofaringe, conforme orientação especializada do Serviço de Controle de Infecção Hospitalar;

- limpeza rigorosa da pele do paciente e tricotomia com máquina no momento da operação;

- uso de campos plásticos iodados aderentes à pele, evitando fixação de compressas na ferida com conseqüentes lesões teciduais;

- uso parcimonioso do eletrocautério na diérese;

- manipulação cuidadosa dos tecidos (48); 
- cuidados com o osso esterno, protegendo-o contra os traumas da serra vibratória de alta energia, abertura exata na linha média do osso, pouco uso de cera, afastador pouco aberto para evitar desvitalização e fraturas, cuidado ao apertar os fios de aço, os quais jamais deverão ser colocados em cartilagem;

- hemostasia rigorosa;

- lavagem freqüente do mediastino com soro fisiológico;

- lavagem da ferida operatória com soro fisiológico no momento do fechamento do subcutâneo;

- controles periódicos dos membros da equipe cirúrgica e anestésica (culturas nasais), assim como de equipamentos utilizados nos centros cirúrgicos;

- manipulação especializada de drenos, cateteres, curativos e da ferida.

A questão do trauma cirúrgico sobre os tecidos, fruto de má técnica operatória, parece ser fundamental na fisiopatologia das infecções mediastinais. Com efeito, alguns cirurgiões chegam ao exagero de incisar a pele com o eletrocautério!

O osso esterno nos parece excessivamente agredido quando se pratica esternotomias. De fato, o osso é violentamente dividido, "cauterizado", preenchido com material estranho (cera de osso), desvascularizado pelas dissecções das artérias torácicas internas e, posteriormente, amarrado fortemente em vários pontos com fios metálicos. Os ortopedistas recomendam cuidados especiais quando se utiliza instrumentos de alta energia em osteotomias e sugerem irrigações constantes com soluções salinas frias que amenizam o trauma derivado do calor. A manipulação cuidadosa do osso esterno pode significar grande diferença para evitar mediastinites (49).

Para fugir da mediastinite e de suas graves conseqüências as seguintes considerações parecem ser pertinentes:

-. Evitar a esternotomia;

- Tomar rigorosas medidas de prevenção;

- Instituir terapia rápida, fazendo-se a rotação do retalho de grande epíploon.

A primeira diz respeito a uma via de acesso que tem sido muito útil, mas que deverá ser realizada cada vez com menor freqüência, dadas as inovações técnicas que a todo momento surgem.

A segunda significa vasculhar pré-operatoriamente os pacientes em busca de focos de infecção, evitar internações prolongadas antes da operação, perseguir a boa técnica durante a manipulação dos tecidos, evitar uso abusivo do eletrocautério, evitar o trauma excessivo do osso esterno, etc.

A terceira, determinar qual a situação clínica em que o paciente se encontra. Caso seja mediastinite, não esperar o processo infeccioso se instalar plenamente, tratar logo, fazendo-se desbridamento e rotação imediata do retalho de epíploon.

RBCCV 44205-483

Sampaio D T, Alves J C R, Silva A F, Lobo Jr. N C, Simões D, Faria W, Lobato A, Figueroa C C S-Mediastinitis after cardiac surgery: treatment using omental flap. Rev Bras Cir Cardiovasc 2000; 15 (1): 23-31.

ABSTRACT: Material and methods: Twenty-two patients with mediastinitis were analyzed out of 1,006 cardiac surgeries with sternotomy performed in "Felício Rocho Hospital" in Belo Horizonte, Minas Gerais, between 1993 and 1998. The complication occurred in 3.2\% patients submitted to Coronary Artery Bypass Grafting (CABG), 3.1\% in transplantations, $1.1 \%$ in valve diseases and none in congenital corrections.

Results: The general infection rate was $2.1 \%$ and the identified was Staphylococcus aureus (39.1\%). Different treatments performed during this period are presented: simple drainage, debridment and rotation of the pectoralis and omental flaps.

Conclusion: The omental flap rotation started on the Service in 1995, considerably improved the results and mortality due to this complication has been eliminated the past 2.5 years.

DESCRIPTORS: Mediastinitis, surgery. Cardiac surgical procedures, adverse effects. Sternum, surgery. Surgical flaps. Omentum, transplantation. 
Sampaio D T, Alves J C R, Silva A F, Lobo Jr. N C, Simões D, Faria W, Lobato A, Figueroa C C S - Mediastinite em cirurgia cardíaca: tratamento com epíploon. Rev Bras Cir Cardiovasc 2000; 15 (1): 23-31.

\section{REFERÊNCIAS BIBLIOGRÁFICAS}

1 Milton H - Mediastinal surgery. Lancet 1897; 1: 872-5.

2 Kirschner M - Tratad de tecnica operatoria general y especial. Barcelona: Editorial Labor 1944: 756-60.

3 Julian O C, Lopez-Belio M, Dye W S - The median sternal incision in intracardiac surgery with extracorporeal circulation: a general evaluation of its use in heart surgery. Surgery 1957; 42: 753-61.

4 Gruntzig A - Transluminal dilatation of coronary-artery stenosis. Lancet 1978; 1: 263. (Letter).

5 Acuff T E, Landreneau R J, Griffith B P, Mack M J Minimally invasive coronary artery bypass grafting. Ann Thorac Surg 1996; 61: 135-7.

6 Benetti F J, Ballester C, Sani G, Doonstra P, Grandjean $\mathrm{J}$ - Video assisted coronary bypass surgery. $J$ Card Surg 1995; 10: 620-5.

7 Berreklouw E \& Alfieri O - Revival of right thoracotomy to approach atrioventricular valves in reoperations. Thorac Cardiovasc Surg 1984; 32: 331-3.

8 Calafiore A M, Giammarco G D, Teodori G et al. Left anterior descending coronary artery grafting via left anterior small thoracotomy without cardiopulmonary bypass. Ann Thorac Surg 1996; 61: 1658-65.

9 Cohn L H, Peigh P S, Sell J, DiSesa V J - Right thoracotomy, femorofemoral bypass, and deep hypothermia for re-replacement of the mitral valve. Ann Thorac Surg 1989; 48: 69-71.

10 Lin $\mathrm{P} \mathrm{J}$, Chang $\mathrm{C} \mathrm{H}$, Chu, $\mathrm{J} \mathrm{J}$ et al. - Video-assisted mitral valve operations. Ann Thorac Surg 1996; 61: 1781-7.

11 Usui A, Kawamura M, Hibi M, Yoshida K, Murakami F, Iwase J - Mitral valve replacement via right thoracotomy after coronary arterial grafting. Ann Thorac Surg 1995; 60: 708-9.

12 Sarr M G, Gott V L, Townsend T R-Mediastinal infection after cardiac surgery. Ann Thorac Surg 1984; 38: 415-23.

13 The Parisian Mediastinitis Study Group - Risk factors for deep sternal wound infection after sternotomy: a prospective, multicenter study. J Thorac Cardiovasc Surg 1996; 111: 1200-7.

14 El Oakley R M \& Wright J E - Postoperative mediastinitis: classification and management. Ann Thorac Surg 1996; 61: 1030-6.

15 Loop F D, Lytle B W, Cosgrove D M et al - Sternal wound complications after isolated coronary artery bypass grafting: early and late mortality, morbidity and cost of care. Ann Thorac Surg 1990; 49: 179-87.

16 Nelson R M \& Dries D J - The economic implications of infection in cardiac surgery. Ann Thorac Surg 1986; 42: $240-6$

17 Culliford A T, Cunningham Jr. J N, Zeff R H, Isom $\mathrm{O}$ W, Teiko $\mathrm{P}$, Spencer F C - Sternal and costochondral infections following open-heart surgery: a review of 2,594 cases. J Thorac Cardiovasc Surg 1976; 72 : 714-26.

Grossi E A, Esposito R, Harris L J et al - Sternal wound infections and use of internal mammary artery grafts. J Thorac Cardiovasc Surg 1991; 102: 342-7.

19 Nishida H, Grooters R K, Soltanzadeh H, Thieman K C - Discriminate use of electrocautery on the median sternotomy incision: a $0.16 \%$ wound infection rate. $J$ Thorac Cardiovasc Surg 1991; 101: 488-94.

20 Nishida H, Grooters R K, Merkley D F, Thieman K C, Soltanzadeh H - Postoperative mediastinitis: a comparison of two electrocautery techniques on presternal soft tissues. J Thorac Cardiovasc Surg 1990; 99: 969-76.

21 Newman L S, Szczukowski L C, Brain R P, Perlino C A - Suppurative mediastinitis after open heart surgery: a case control study of risk factors. Chest 1988; 94: 546-53.

Bitkover C Y \& Garldlund B - Mediastinitis after cardiovascular operations: a case-control study of risk factors. Ann Thorac Surg 1998; 65: 36-40.

Arnold M - The surgical anatomy of sternal blood supply. $J$ Thorac Cardiovasc Surg 1972; 64: 596-610.

24 Breyer R H, Mills S A, Hudspeth A S, Johnston F R, Cordell A R - A prospective study of sternal wound complications. Ann Thorac Surg 1984; 37: 412-6.

25 Madden J E, Edlich R F, Custer J R, Panek P H, Thul $\mathrm{J}$, Wangensteen $\mathrm{O} \mathrm{H}$ - Studies in the management of the contaminated wound. VI - Resistance to infection of surgical wounds made by knife, electrosurgery, and laser. Am J Surg 1970; 119: 222 4.

26 Ottino G, De Paulis R, Pansini S et al. - Major sternal wound infection after open-heart surgery: a multivariate analysis of risk factors in 2,579 consecutive operative procedures. Ann Thorac Surg 1987; 44: 173-9.

27 Calvat S, Trouillet J L, Nataf P, Vuagnat A, Chastre J, Gilbert C - Closed drainage using Redon catheters for local treatment of poststernotomy mediastinitis. Ann Thorac Surg 1996; 61: 195-201.
28

29
Molina J E - Primary closure for infected dehiscence of the sternum. Ann Thorac Surg 1993; 55: 459-63.

Shumacker Jr. H B \& Mandelbaum I - Continuous antibiotic irrigation in the treatment of infection. Arch Surg 1963; 86: 54-7.

Acinapura A J, Godfrey N, Romita M et al. - Surgical 
Sampaio D T, Alves J C R, Silva A F, Lobo Jr. N C, Simões D, Faria W, Lobato A, Figueroa C C S - Mediastinite em cirurgia cardíaca: tratamento com epíploon. Rev Bras Cir Cardiovasc 2000; 15 (1): 23-31.

management of infected median sternotomy: closed irrigation vs. muscle flaps. J Cardiovasc Surg 1985; 26: 443-6.

31 Arnold P G \& Pairolero P C - Chest-wall reconstruction: an account of 500 consecutive patients. Plast Reconstr Surg 1996; 98: 804-10.

Belcher P, McLean N, Breach N, Paneth M - Omental transfer in acute and chronic sternotomy wound breakdown. Thorac Cardiovasc Surg 1990; 38: 186-91.

Grossi E A, Culliford A T, Krieger K H et al. - A survey of 77 major infectious complications of median sternotomy: a review of 7,949 consecutive operative procedures. Ann Thorac Surg 1985; 40: 214-23.

Jeevanandam V, Smith C R, Rose E A, Malm J R, Hugo $\mathrm{N} E$ - Single-stage management of sternal wound infections. J Thorac Cardiovasc Surg 1990; 99: 256-63.

Jurkiewicz M J \& Arnold P G - The omentum: an account of its use in the reconstruction of the chest wall. Ann Surg 1977; 185: 548-54.

Lee Jr. A B, Schimert G, Shaktin S, Seigel J H - Total excision of the sternum and thoracic pedicle transposition of the greater omentum: useful strategems in managing severe mediastinal infection following open heart surgery. Surgery 1976; 80: 433-6.

37 Pairolero P C \& Arnold P G - Management of recalcitrant median sternotomy wounds. J Thorac Cardiovasc Surg 1984; 88: 357-64.

Scully H E, Leclerc Y, Martin R D et al. - Comparison between antibiotic irrigation and mobilization of pectoral muscle flaps in treatment of deep sternal infections. $J$ Thorac Cardiovasc Surg 1985; 90: 523-31.

Prevost L G, Subramainian V A, Rothaus K O, Dineen $P$ - A comparison of the open and closed methods in the initial treatment of sternal wound infections. $J$ Cardiovasc Surg 1989; 30: 757-63.
Kiricuta I - L'emploi du grand épiplon dans la chirugie du sein cancéreux. Presse Med (Paris) 1963; 71: 15.

41 O'Shaughnessy L - Surgical treatment of cardiac ischaemia. Lancet 1937; 1: 185- 94.

42 Cartier R, Brunette I, Hashimoto K, Bourne W M, Schaff $\mathrm{H} \mathrm{V} \mathrm{-} \mathrm{Angiogenic} \mathrm{factor:} \mathrm{a} \mathrm{possible} \mathrm{mechanism} \mathrm{for}$ neovascularization produced by omental pedicles. $J$ Thorac Cardiovasc Surg 1990; 99: 264-8.

43 Lopez-Monjardin H, de-la-Pena-Salcedo A, Mendoza Munhoz M, Lopez-Yanez-de-la-Pena A, Palacio-Lopez E, Lopez-Garcia A - Omentum flap versus pectoralis major flap in the treatment of mediastinitis. Plast Reconstr Surg 1998; 101: 1481-5.

44 Telfer J R, Chapple D C, Powell B W - Metastatic colonic adenocarcinoma in a pedicled omental flap used for sternal reconstruction: a case report. Br J Plast Surg 1996; 49: 67-9.

45 Sinatra R S - Acute pain: mechanisms \& management. USA: Mosby-Year Book, 1992: 46-57.

46 Ochsner J L, Mills N L, Woolverton W C - Disruption and infection of the median sternotomy incision. $J$ Cardiovasc Surg 1972; 13: 394-9.

47 Stoney W S, Alford Jr. W C, Burrus G R, Frist R A, Thomas Jr. C S - Median sternotomy dehiscence. Ann Thorac Surg 1978; 26: 421-6.

48 Ferguson D J-Advances in the management of surgical wounds. Surg Clin North Am 1971; 51: 49-59.

49 Timmes $\mathrm{J} \mathrm{J}$, Wolvek S, Fernando M, Bas M, Rocko J - A new method of sternal approximation. Ann Thorac Surg 1973; 15: 544-6.

50 Milano C A, Georgiade G, Muhlbaier L H, Smith P K, Wolfe W G - Comparison of omental and pectoralis flaps for poststernotomy mediastinitis. Ann Thorac Surg 1999; 67: 377-81. 